\title{
Should simultaneous stoma closure and incisional hernia repair be avoided?
}

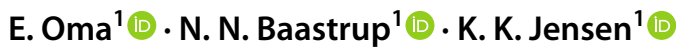

Received: 14 July 2020 / Accepted: 17 September 2020 / Published online: 25 September 2020

c) Springer-Verlag France SAS, part of Springer Nature 2020

\begin{abstract}
Purpose Patients scheduled for stoma closure may also have an incisional hernia. Studies have reported acceptable outcomes after contaminated ventral hernia repair, but whether stoma closure and incisional hernia repair should be performed as a combined procedure is unknown. This study examined combined stoma closure and incisional hernia repair compared with incisional hernia repair only.

Methods This was a nationwide propensity-score matched study. Patients who underwent elective incisional hernia repair from 2007-2017 were identified in the Danish Hernia Database. All patients who underwent concurrent stoma closure were matched 1:3 with patients who underwent incisional hernia repair only. The primary outcome was reoperation for hernia recurrence, whereas secondary outcomes included anastomotic leakage, length of hospital stay, and 30-day reoperation and readmission rates.

Results In total, 516 patients were included. The risk of reoperation for recurrence was increased after concurrent stoma closure compared with incisional hernia repair only (hazard ratio $1.69,95 \%$ confidence interval $1.01-2.82, p=0.044$ ). Seven (5.4\%) patients who underwent incisional hernia repair concurrent to stoma closure were reoperated for anastomotic leakage. Length of hospital stay and reoperation rates within 30 days were increased after concurrent stoma closure compared with incisional hernia repair only (median 8 versus 3 days, $p<0.001$ and $29.5 \%$ versus $18.6 \%, p=0.013$ ), whereas there was no difference in 30-day readmission rates $(p=0.251)$.

Conclusions Stoma closure and incisional hernia repair should be performed as a dual-stage procedure to decrease the risk of hernia recurrence.
\end{abstract}

Keywords Stoma reversal $\cdot$ Incisional hernia repair $\cdot$ Ventral hernia repair $\cdot$ Hernia recurrence

\section{Introduction}

A temporary stoma is indicated when a primary anastomosis is considered dangerous or to protect a high-risk anastomosis [1]. Following laparotomy, up to $22 \%$ of patients develop an incisional hernia [2]. For patients scheduled for stoma closure with a coexisting incisional hernia, concurrent stoma closure and hernia repair or a two-stage approach are the

The manuscript was accepted as a podium presentation at the $42^{\text {nd }}$ Annual European Hernia Society Congress, Barcelona, Spain, 4-6 May 2020 (Congress cancelled due to COVID-19).

E. Oma

erlingom@gmail.com

1 Digestive Disease Center, Bispebjerg Hospital, Bispebjerg Bakke 23, 2400 Copenhagen, NV, Denmark options. A single-stage concurrent procedure may have the advantage of avoiding an additional surgical procedure with its inherent risk of complications and added recovery. However, prolonged operative duration and contamination might increase the risk of short-term morbidity and potentially hernia recurrence, compared with a dual-stage procedure with a delayed definitive hernia repair [3, 4].

This study aimed to examine outcomes following incisional hernia repair with and without concurrent stoma closure. The primary outcome of the study was reoperation for hernia recurrence, and the secondary outcomes were the length of hospital stay (LOS), overall reoperation rates within 30 days, reoperation rates for anastomotic leakage, and readmission within 30 days. 


\section{Methods}

This was a nationwide, propensity-score matched study reported according to the STROBE statement [5]. All patients who underwent elective incisional hernia repair between 1 January 2007 and 31 December 2017 were identified in the Danish Ventral Hernia Database (DVHD). This registry contains prospectively registered data on all patients undergoing ventral hernia repair in Denmark and includes patient demographics, hernia characteristics, and technical aspects of the surgical repair [6]. Data was merged with the Danish National Patient Registry (DNPR) to identify patients who underwent stoma closure concurrent to the incisional hernia repair, using the unique Danish personal registration number. The DNPR is a complete nationwide registry with data on procedures and diagnoses from hospitals in Denmark [7]. Concurrent stoma closure was defined as registration of a procedure code for stoma closure (KJFG00 through KJFG47 [Nordic Medico-Statistical Committee Classification of Surgical Procedures [8]]) on the same date as the incisional hernia repair registered in the DVHD. Parastomal hernia repair is registered as its own entity in the DVHD and was not included in the study. Thus, patients who underwent concurrent stoma closure had an incisional hernia remote from the stoma site.

The final study cohort included patients who underwent stoma closure concurrent to incisional hernia repair and a 1:3 propensity-score matched control group of patients who underwent incisional hernia repair only. The primary outcome was reoperation for hernia recurrence, as registered in the DVHD. Secondary outcomes included length of postoperative hospital stay (LOS), overall reoperation rates within 30 days, reoperation rates for anastomotic leakage (procedure code KJWF00), and readmission within 30 days, as registered in the DNPR.

\section{Statistics}

Propensity-score matching was performed with a logistic regression model using the nearest neighbor method without replacement and a 0.2 caliper width $[9,10]$. Matching variables included age, sex, transverse defect size, surgical approach (open, laparoscopic or converted), and repair technique (mesh or suture repair). The DVHD includes data on whether any other concurrent procedures were performed (without further specification), and accordingly, only patients registered as undergoing nothing but incisional hernia repair, were considered as potential controls. Baseline characteristics were compared with the Wilcoxon rank-sum test and $\chi^{2}$ test for continuous and categorical variables, respectively. The risk of reoperation for hernia recurrence was analyzed with Cox proportional hazards regression analysis and reported as a hazard ratio (HR) with 95\% confident intervals (CI). Follow-up was from the initial repair until reoperation for hernia recurrence, death or 31 December 2018, and calculated as median (interquartile range [IQR]) with the reverse Kaplan Meier method [11]. LOS was compared between groups with Mann Whitney $\mathrm{U}$, whereas the risk of reoperation and readmission was compared with $\chi^{2}$ test. Analyses were performed with R v. 3.5.2. (R Foundation for Statistical Computing, R-project.org). The study was approved by the Danish Data Protection Agency (ref. BFH-2012-580004). Ethical approval nor informed consent was required according to Danish legislation.

\section{Results}

In total, 6,638 patients underwent elective incisional hernia repair during the inclusion period. Of these, $129(1.9 \%)$ underwent incisional hernia repair concurrent to stoma closure and were matched with 387 patients who underwent incisional hernia repair only (Table 1 ). The median followup was 6.2 years (IQR 4.3-8.6 years). A mesh repair was performed in $393(76.2 \%)$ patients. The median transverse

Table 1 Baseline characteristics of included patients who underwent elective incisional hernia repair with or without concurrent stoma closure, $N=516$

\begin{tabular}{llll}
\hline Variable & $\begin{array}{l}\text { Concurrent } \\
\text { stoma closure } \\
N=129\end{array}$ & $\begin{array}{l}\text { Incisional hernia } \\
\text { repair only } \\
N=387\end{array}$ & \\
\hline Age, years & $62(52-68)$ & $63(51-71)$ & 0.180 \\
Sex & & & 0.914 \\
Female & $42(32.6)$ & $130(33.6)$ & \\
Male & $87(67.4)$ & $257(66.4)$ & \\
Hernia defect width, cm & $10(5-12)$ & $8(4-15)$ & 0.824 \\
Surgical approach & & & 0.850 \\
Converted & $1(0.8)$ & $4(1.0)$ & \\
Laparoscopic & $12(9.3)$ & $42(10.9)$ & \\
Open & $116(89.9)$ & $341(88.1)$ & \\
Use of mesh & $94(72.9)$ & $299(77.3)$ & 0.371 \\
Type of stoma & & & \\
Ileostomy & $64(49.6)$ & - & \\
Colostomy & $65(50.4)$ & - & \\
Type of anastomosis & & & \\
Ileo-ileal & $36(27.9)$ & - & \\
Ileo-colonic & $25(19.4)$ & - & \\
Ileo-rectal & $3(2.3)$ & - & \\
Colo-colonic & $28(21.7)$ & - & \\
Colo-rectal & $37(28.7)$ & - & \\
\hline
\end{tabular}

Numbers are median (interquartile range) or $N(\%)$ 
defect size was $10 \mathrm{~cm}$ (IQR 6-15 cm) in patients who had mesh reinforcement compared with $3 \mathrm{~cm}$ (IQR $1-10 \mathrm{~cm}$ ) in patients who underwent a sutured repair $(p<0.001)$. Sixtyfour (49.6\%) patients underwent ileostomy closure and 65 (50.4\%) patients underwent colostomy closure.

\section{Reoperation for hernia recurrence}

Twenty-three (17.8\%) and $41(10.6 \%)$ patients underwent reoperation for hernia recurrence after incisional hernia repair concurrent to stoma closure and incisional hernia repair only, respectively. The median time to reoperation for hernia recurrence was 1.6 years (IQR $0.8-2.6$ years). The risk of reoperation for hernia recurrence was significantly increased after incisional hernia repair concurrent to stoma closure compared with incisional hernia repair only (HR 1.69, 95\% CI 1.01-2.82, $p=0.044$ ). A multivariable model adjusting for the matching variables showed similar results (HR 1.72, 95\% CI 1.03-2.87, $p=0.039$ ). The fiveyear cumulative incidence of reoperation for recurrence was $15.9 \%$ (95\% CI 9.5-22.3\%) and 9.6\% (6.6-12.6\%) after incisional hernia repair concurrent to stoma closure and incisional hernia repair only, respectively. The cumulative incidence of reoperation for hernia recurrence is shown in Fig. 1. Compared with the full cohort, similar results were found in a subgroup univariable analysis of patients who had mesh reinforcement (HR for reoperation for hernia recurrence after concurrent procedure versus incisional hernia repair only $1.54,95 \%$ CI $0.85-2.79, p=0.159)$. In a subgroup analysis of patients who underwent incisional hernia repair concurrent to stoma closure, there was a nonsignificant increased risk of reoperation for recurrence following ileostomy vs. colostomy closure (HR 1.89, $95 \%$ CI $0.79-4.55, p=0.153$ ).

\section{Secondary outcomes}

LOS was significantly increased for patients who underwent incisional hernia repair concurrent to stoma closure compared with incisional hernia repair only [median 8 days (IQR $5-12$ days) versus 3 days ( $1-7$ days), $p<0.001]$. Thirty-eight (29.5\%) patients underwent reoperation within 30 days after incisional hernia repair concurrent to stoma closure compared with 72 (18.6\%) patients after incisional hernia repair only ( $p=0.013$, Table 2 ). In a multivariable analysis, the risk of reoperation for hernia recurrence was increased in patients who underwent reoperation within 30 days compared to patients who did not (HR 2.04, 95\% CI 1.17-3.52, $p=0.011)$. Seven (5.4\%) patients who underwent incisional hernia repair concurrent to stoma closure were reoperated for anastomotic leakage. Of these, two patients subsequently underwent reoperation for hernia recurrence. There was no difference comparing stoma type and anastomosis type on the risk of anastomotic leakage ( $p=0.425$ and $p=0.610$, respectively). There was no difference in readmission rates within 30 days for patients who underwent incisional hernia repair concurrent to stoma closure compared with incisional hernia repair only $(p=0.251)$.
Fig. 1 Cumulative incidence of reoperation for hernia recurrence after incisional hernia repair with and without concurrent stoma closure

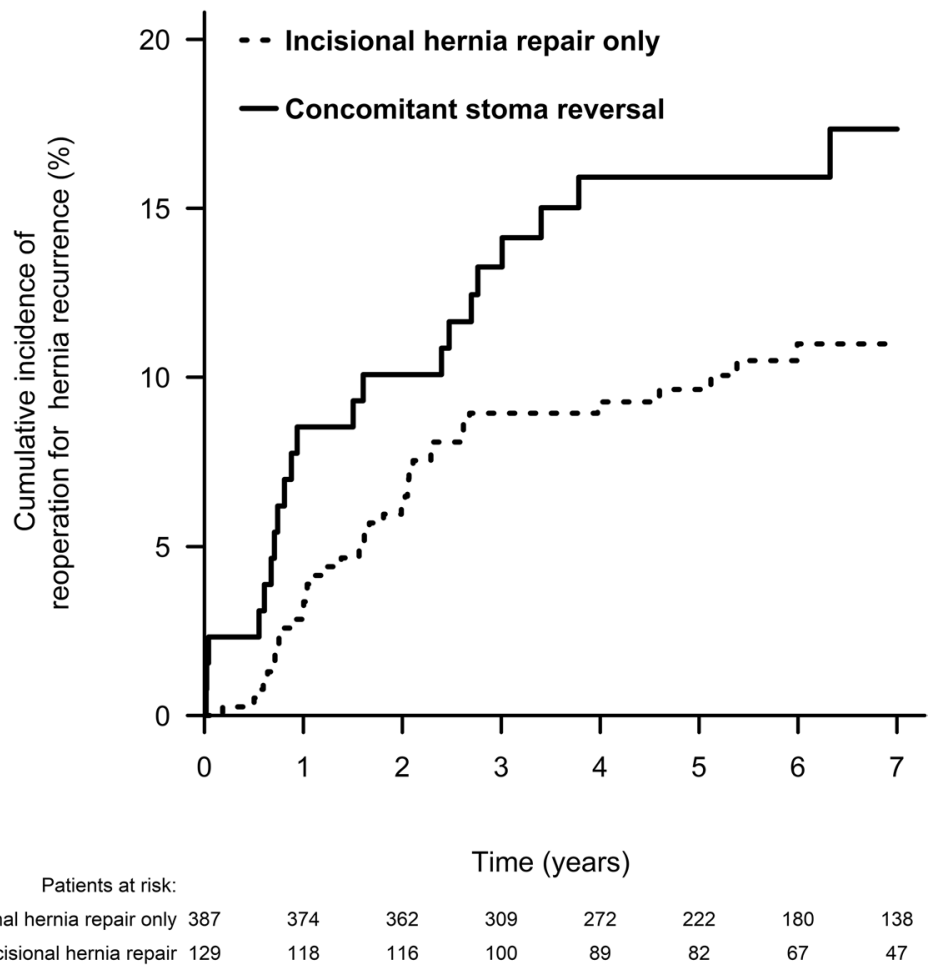


Table 2 Short-term outcomes after elective incisional hernia repair with or without concurrent stoma closure, $N=516$

\begin{tabular}{llll}
\hline Variable & $\begin{array}{l}\text { Concomitant stoma } \\
\text { reversal } \\
N=129\end{array}$ & $\begin{array}{l}\text { Incisional hernia repair } \\
\text { alone } \\
N=387\end{array}$ & $p$ \\
\hline Length of stay, days & $8(5-12)$ & $3(1-7)$ & $<0.001$ \\
30-day reoperation rate & $38(29.5)$ & $72(18.6)$ & 0.013 \\
Explorative laparotomy & $1(0.8)$ & $3(0.8)$ & \\
Superficial surgical site infection & $12(9.3)$ & $30(7.8)$ & \\
Deep surgical site infection & $7(5.4)$ & $4(1.0)$ & \\
Deep bleeding & $3(2.3)$ & $2(0.5)$ & \\
Bowel resection & $3(2.3)$ & $2(0.0)$ & 0.251 \\
Small bowel obstruction & $0(0.0)$ & $31(8.0)$ & $98(25.3)$ \\
Other & $12(9.3)$ & & \\
30-day readmission rate & $40(31.0)$ & & \\
\hline
\end{tabular}

Numbers are median (interquartile range) or $N(\%)$

${ }^{a}$ Including reoperation for anastomotic leakage

\section{Sensitivity analyses}

To assess the impact of anastomotic leakage, additional analyses were performed excluding the seven patients who had anastomotic leakage after a concomitant procedure (122 patients compared with 387 patients with incisional hernia repair only). Compared with incisional hernia repair only, LOS was significantly increased after concurrent stoma closure (median 8 and 3 days, $p<0.001$ ). There was no significant difference in reoperation rates within 30 days $(31 / 122=25.4 \%$ versus $72 / 387=18.6 \%$, $p=0.133$ ). The rates of reoperation for hernia recurrence were $21 / 122=17.2 \%$ and $41 / 387=10.6 \%$, respectively. In a multivariable Cox regression analysis, there was no significant difference between the groups (concomitant procedure vs incisional hernia repair only HR $1.6295 \%$ CI $0.96-2.75$, $p=0.074)$.

\section{Discussion}

In the current study, concurrent stoma closure was associated with an increased risk of reoperation for hernia recurrence and increased LOS and risk of reoperation within 30 days after incisional hernia repair. This is the first study to examine hernia-related outcomes following incisional hernia repair with or without concurrent stoma closure.

Studies have reported acceptable outcomes following the repair of contaminated ventral hernias [12-14]. However, the risk of wound morbidity and hernia recurrence increase with the severity of contamination, in particular dirty/infected wounds (CDC wound class 4) [15-18]. The results of the current study indicate that the risk of hernia recurrence is significantly increased after incisional hernia repair with a clean-contaminated versus clean wound (CDC wound class
1 versus 2), representing the common circumstances for elective incisional hernia repair concurrent to stoma closure and elective incisional hernia repair only. Apart from the associated wound class, other factors reflecting the increased complexity of a concurrent procedure might also affect the risk of recurrence.

Consistent with the present findings, increased financial costs for combined ventral hernia repair and stoma closure compared with two separate procedures have been reported, thus advocating for a dual-stage approach from an economical point-of-view [19]. In the present study, data on a stoma closure prior to an incisional hernia repair was not included. Thus, more studies are needed to grasp all clinical consequences of a single- versus dual-stage procedure for the management of a temporal stoma and coexistent incisional hernia. Not surprisingly, the combined procedure was associated with an increased LOS. For a combined procedure, the limiting factor for discharge will typically be the assurance of anastomosis viability. LOS after a compared procedure should ideally be compared with both hospital stays of a staged procedure. A previous study examined concurrent incisional hernia repair and stoma closure compared with stoma closure only [20]. The study found an increased risk of anastomotic leakage requiring surgery in patients undergoing concurrent incisional hernia repair (22\% versus $2.4 \%$ after stoma closure only, $p=0.002$ ). Contrary, in this larger study, only $5.4 \%$ of patients who underwent incisional hernia repair concurrent to stoma closure underwent reoperation for anastomotic leakage. Thus, adding incisional hernia repair to stoma closure does not seem to have a substantial impact on anastomotic failure rates.

Some patients undergoing a combined stoma closure and hernia repair may have been reoperated for hernia recurrence at the stoma closure site, as the precise location of the operated incisional hernia is not registered in the DHDB. 
Incisional hernia formation at the stoma closure site is reported in up to $36 \%$ of patients [21, 22]. Prophylactic mesh-reinforcement at the stoma closure site is an option for a single-stage procedure, to decrease the risk of a stoma closure-site hernia [23].

In this study, $27.1 \%$ of the patients with a concurrent procedure underwent a sutured incisional hernia repair. Suture repair was performed for small defects (median $3 \mathrm{~cm}$ transverse defect size); however, a mesh is recommended for incisional hernia repair, irrespective of defect size, to reduce the risk of recurrence [24]. Reasons for omitting mesh reinforcement in the current study were not available. Although all patients were registered as undergoing incisional hernia repair in the DHDB, a concurrent procedure without mesh might not have been considered a devoted single-stage procedure by the operating surgeon. This might have biased the results, albeit mesh or suture repair was one of the matching variables. However, compared with the full cohort, similar results were found in a subgroup analysis of mesh repairs only, although without reaching statistical significance. If the hernia does not recur, the patient is spared from additional surgery. In case of recurrence, reoperation after a suture repair is typically not more complicated compared with the previous repair. However, if a more extensive hernia repair was performed concomitant to stoma closure, e.g. including component separation, a reoperation for hernia recurrence might be overly complicated as the intended surgical planes may be difficult to enter, i.e., "burned bridge" [4].

This study had several limitations. Although propensityscore matching was performed, there was a risk of selection bias due to the retrospective observational study design. Data on important predictors of hernia recurrence and anastomotic leakages such as body mass index, use of tobacco and diabetes mellitus was not available. The incisional hernia was not registered according to the European Hernia Society classification, as this information was not part of the Danish Hernia Database at the time of our study [25]. In the current study, only surgical site occurrences requiring surgical reintervention were included since conservatively managed complications are not part of the Danish Hernia Database. Thus, the true incidence of surgical site occurrences after incisional hernia repair with and without concomitant stoma closure in this study remains unknown. Further, the Danish Hernia Database has no data on symptoms related to the incisional hernia or quality of life, neither pre- or postoperatively. Therefore, we have no information about any hypothetical differences in patient-reported outcomes after incisional hernia repair with or without concomitant stoma closure. For patients undergoing concurrent stoma closure and incisional hernia repair, the time from stoma creation was not available. Furthermore, mesh type and placement and reasons for readmission within 30 days were not uniformly registered and thus not available for analysis. Strengths of the study include the use of nationwide data with a complete follow-up, constituting high external validity.

Concurrent incisional hernia repair and stoma closure led to worse outcomes compared with incisional hernia repair only. It seems that anastomotic leakage was a substantial factor for worse outcomes in the concomitant procedure group. The decision to perform a single- or dual-stage approach should be carefully evaluated and discussed with each individual patient. In patients with a low risk of anastomotic leakage and other complications, a single-stage approach might be preferred, as an additional surgical procedure seems avoidable in a majority of patients. However, based on these findings, it is suggested that stoma closure and incisional hernia repair should be performed as a dual-stage procedure to decrease the risk of hernia recurrence.

Data availability Disaggregated data used in this study are available on request.

\section{Compliance with ethical standards}

Conflict of interest All authors declare no conflict of interest.

Ethics approval This study did not need approval from an ethics committee according to Danish law.

Human and animal rights This article does not contain any studies with human participants or animals performed by any of the authors.

Informed consent Informed consent was not required for this study according to Danish law.

\section{References}

1. Tan WS, Tang CL, Shi L, Eu KW (2009) Meta-analysis of defunctioning stomas in low anterior resection for rectal cancer. Br J Surg 96:462-472. https://doi.org/10.1002/bjs.6594

2. Fink C, Baumann P, Wente MN, Knebel P, Bruckner T, Ulrich A, Werner J, Buchler MW, Diener MK (2014) Incisional hernia rate 3 years after midline laparotomy. Br J Surg 101:51-54. https://doi. org/10.1002/bjs.9364

3. Heniford BT, Ross SW, Wormer BA, Walters AL, Lincourt AE, Colavita PD, Kercher KW, Augenstein VA (2018) Preperitoneal ventral hernia repair: a decade long prospective observational study with analysis of 1023 patient outcomes. Ann Surg. https:// doi.org/10.1097/SLA.0000000000002966

4. Petro CC, Rosen MJ (2018) Fight or flight: the role of staged approaches to complex abdominal wall reconstruction. Plast Reconstr Surg 142:38S-44S. https://doi.org/10.1097/PRS.00000 00000004847

5. von Elm E, Altman DG, Egger M, Pocock SJ, Gotzsche PC, Vandenbroucke JP, Initiative S (2007) The strengthening the reporting of observational studies in epidemiology (STROBE) statement: guidelines for reporting observational studies. Lancet 370:14531457. https://doi.org/10.1016/S0140-6736(07)61602-X 
6. Helgstrand F, Jorgensen LN (2016) The Danish ventral hernia database: a valuable tool for quality assessment and research. Clin Epidemiol 8:719-723. https://doi.org/10.2147/CLEP.S99501

7. Lynge E, Sandegaard JL, Rebolj M (2011) The Danish national patient register. Scand J Pub Health 39(7):30-33. https://doi. org/10.1177/1403494811401482

8. Committee NM-S (2010) NOMESCO classification of surgical procedures (NCSP). https://norden.diva-portal.org/smash/get/ diva2:970547/FULLTEXT01.pdf. Accessed 24 November 2018

9. Austin PC (2011) Optimal caliper widths for propensity-score matching when estimating differences in means and differences in proportions in observational studies. Pharm Stat 10:150-161. https://doi.org/10.1002/pst.433

10. Lonjon G, Porcher R, Ergina P, Fouet M, Boutron I (2017) Potential pitfalls of reporting and bias in observational studies with propensity score analysis assessing a surgical procedure: a methodological systematic review. Ann Surg 265:901-909. https://doi. org/10.1097/SLA.0000000000001797

11. Schemper M, Smith TL (1996) A note on quantifying follow-up in studies of failure time. Control Clin Trials 17:343-346. https ://doi.org/10.1016/0197-2456(96)00075-x

12. de Vries FEE, Hodgkinson JD, Claessen JJM, van Ruler O, Leo CA, Maeda Y, Lapid O, Obdeijn MC, Tanis PJ, Bemelman WA, Constantinides J, Hanna GB, Warusavitarne J, Vaizey C, Boermeester MA (2020) Long-term outcomes after contaminated complex abdominal wall reconstruction. Hernia. https://doi. org/10.1007/s 10029-020-02124-7

13. Majumder A, Winder JS, Wen Y, Pauli EM, Belyansky I, Novitsky YW (2016) Comparative analysis of biologic versus synthetic mesh outcomes in contaminated hernia repairs. Surgery 160:828838. https://doi.org/10.1016/j.surg.2016.04.041

14. Warren J, Desai SS, Boswell ND, Hancock BH, Abbad H, Ewing JA, Carbonell AM, Cobb WS (2020) Safety and efficacy of synthetic mesh for ventral hernia repair in a contaminated field. J Am Coll Surg. https://doi.org/10.1016/j.jamcollsurg.2019.12.008

15. Cobb WS, Warren JA, Ewing JA, Burnikel A, Merchant M, Carbonell AM (2015) Open retromuscular mesh repair of complex incisional hernia: predictors of wound events and recurrence. J Am Coll Surg 220:606-613. https://doi.org/10.1016/j.jamcollsur g.2014.12.055

16. Tubre DJ, Schroeder AD, Estes J, Eisenga J, Fitzgibbons RJ Jr (2018) Surgical site infection: the "Achilles Heel" of all types of abdominal wall hernia reconstruction. Hernia 22:1003-1013. https ://doi.org/10.1007/s10029-018-1826-9

17. Ortega G, Rhee DS, Papandria DJ, Yang J, Ibrahim AM, Shore AD, Makary MA, Abdullah F (2012) An evaluation of surgical site infections by wound classification system using the
ACS-NSQIP. J Surg Res 174:33-38. https://doi.org/10.1016/j. jss.2011.05.056

18. Berger RL, Li LT, Hicks SC, Davila JA, Kao LS, Liang MK (2013) Development and validation of a risk-stratification score for surgical site occurrence and surgical site infection after open ventral hernia repair. J Am Coll Surg 217:974-982. https://doi. org/10.1016/j.jamcollsurg.2013.08.003

19. Madabhushi V, Plymale MA, Roth JS, Johnson S, Wade A, Davenport DL (2018) Concomitant open ventral hernia repair: what is the financial impact of performing open ventral hernia with other abdominal procedures concomitantly? Surg Endosc 32:1915-1922. https://doi.org/10.1007/s00464-017-5884-3

20. Baastrup NN, Hartwig MFS, Krarup PM, Jorgensen LN, Jensen KK (2019) Anastomotic leakage after stoma reversal combined with incisional hernia repair. World J Surg 43:988-997. https:// doi.org/10.1007/s00268-018-4866-5

21. Lambrichts DPV, de Smet GHJ, van der Bogt RD, Kroese LF, Menon AG, Jeekel J, Kleinrensink GJ, Lange JF (2018) Incidence, risk factors and prevention of stoma site incisional hernias: a systematic review and meta-analysis. Colorectal Dis 20:O288-O303. https://doi.org/10.1111/codi.14369

22. Mongelard K, Mynster T, Jensen KK (2020) Stoma-site hernia after stoma reversal following rectal cancer resection. Dan Med J 67(3):A06190353

23. van den Hil LCL, van Steensel S, Schreinemacher MHF, Bouvy ND (2019) Prophylactic mesh placement to avoid incisional hernias after stoma reversal: a systematic review and meta-analysis. Hernia 23:733-741. https://doi.org/10.1007/s10029-019-01996-8

24. Luijendijk RW, Hop WC, van den Tol MP, de Lange DC, Braaksma MM, JN IJ, Boelhouwer RU, de Vries BC, Salu MK, Wereldsma JC, Bruijninckx CM, Jeekel J (2000) A comparison of suture repair with mesh repair for incisional hernia. N Engl J Med 343:392-398. https://doi.org/10.1056/NEJM200008103430603

25. Muysoms FE, Miserez M, Berrevoet F, Campanelli G, Champault GG, Chelala E, Dietz UA, Eker HH, El Nakadi I, Hauters P, Hidalgo Pascual M, Hoeferlin A, Klinge U, Montgomery A, Simmermacher RK, Simons MP, Smietanski M, Sommeling C, Tollens T, Vierendeels T, Kingsnorth A (2009) Classification of primary and incisional abdominal wall hernias. Hernia 13:407414. https://doi.org/10.1007/s10029-009-0518-x

Publisher's Note Springer Nature remains neutral with regard to jurisdictional claims in published maps and institutional affiliations. 